\title{
Autosolitons in trapped Bose-Einstein condensates with two- and three-body inelastic processes
}

\author{
Victo S. Filho, ${ }^{1}$ F. Kh. Abdullaev, ${ }^{1,2}$ A. Gammal, ${ }^{1}$ and Lauro Tomio ${ }^{1}$ \\ ${ }^{1}$ Instituto de Física Teórica, Universidade Estadual Paulista, 01405-900, São Paulo, Brazil \\ ${ }^{2}$ Physical-Technical Institute, Tashkent, Uzbekistan \\ (Received 10 October 2000; published 13 April 2001)
}

\begin{abstract}
In this paper, we consider the conditions for the existence of autosolitons, in trapped Bose-Einstein condensates with attractive atomic interactions. First, the variational approach is employed to estimate the stationary solutions for the three-dimensional Gross-Pitaevskii equation with trap potential, linear atomic feeding from the thermal cloud, and two- and three-body inelastic processes. Next, by using exact numerical calculations, we show that the variational approach gives reliable analytical results. We also discuss the possible observation of autosolitons in experiments with ${ }^{7} \mathrm{Li}$.
\end{abstract}

DOI: 10.1103/PhysRevA.63.053603

\section{INTRODUCTION}

The existence of envelope autosolitons in the onedimensional (1D) case, in a homogeneous nonlinear medium with dissipation and amplification, was revealed by Pereira and Stenflo [1]. They found the exact solution for autosolitons with arbitrary growth and damping strengths in the perturbed nonlinear Schrödinger equation (NLSE). Later, autosolitons were discovered in nonlinear fiber optics, namely, in fibers with amplifiers and distributed filters (the latter corresponds to the frequency-dependent damping in the nonlinear Schrödinger equation) $[2,3]$ and also for waves on the surface of deep water [4]. Correspondingly, in a 2D homogeneous medium with amplification and nonlinear damping, the possibility of existence of a 2D analog of the PereiraStenflo solitons was recently shown by a variational approach [5]. Autosolitons in a weakly dispersive nonlinear media, described by the Korteweg-deVries equation, have been studied in Refs. [6,7].

The autosolitons can be distinguished from ordinary solitons. The latter exist in a conservative media and are originated from the balance between the nonlinear and dispersive effects of the wave propagation. The properties of these generated solitons are defined by the initial conditions (their number, parameters such as amplitudes, widths, etc.) [6], with the solutions characterized by their corresponding properties. As for to the autosolitons, they can be generated in nonconservative media when effects of amplification and dissipation are present. For the existence of autosolitons, one should add to the equilibrium condition between nonlinearity and dispersion the requirement of a balance between amplification, frequency-dependent damping, and nonlinear dissipation. In distinction from ordinary solitons, the properties of the autosoliton, as a rule, are fixed by the coefficients of the perturbed NLSE and by any initial perturbation that is attracted to this point (attractor in the space of coefficients). Mathematically, the problem is described by the NLSE with complex parameters. If the imaginary parts are large, the equation is equivalent to the so-called complex GinzburgLandau equation. An interesting limit is represented by the NLSE with small complex coefficients.

The purpose of this paper is to show that the analog of the autosoliton is possible in a trapped Bose-Einstein condensate (BEC). Recently the existence of bright and dark solitons in
PACS number(s): 03.75.Fi, 32.80.Pj, 42.50.Md, 42.81.Dp

BEC has been demonstrated (see theory in $[8,9]$ ). Dark solitons have already been observed in BEC with repulsive interaction between atoms [10]. For attractive interactions, bright solitons exist in 1D BEC. It is well known that twoand three-dimensional condensates with attractive interaction between atoms and trapping potential are unstable if the number of atoms exceeds the critical value $\left(N_{c}\right)$. Below this value a stable ground state can exist, corresponding to solitary solutions $[11,12]$. When the number of atoms exceeds $N_{c}$ the collapse occurs. At large densities the inelasticscattering processes involving two and three atoms come into play, leading to the effective nonlinear damping of the condensate. The process of feeding atoms from the thermal cloud can be modeled as a linear amplification described in Ref. [13], where the relevance of the three-body inelastic processes was discussed. The statistical data obtained from experiments with ${ }^{7} \mathrm{Li}$ supports the growing and collapse picture $[14,15]$.

Numerical simulations of the 3D Gross-Pitaevskii (GP) equation are performed in Refs. $[13,16]$. The present paper shows that periodic oscillations occur in the condensate and, for particular values of atomic feeding and three-body dissipation parameters, stable states of the cloud can exist. Thus, we can expect the occurrence of analog autosolitons in 2D and 3D BEC's. The problem is described by the complex Ginzburg-Landau equation with trapping potential in the NLSE limit with small nonconservative perturbations. This equation is nonintegrable and, therefore, analytical solutions can only be obtained by considering approximate methods like the variational approach [17]. In the following, we first use the time-dependent variational approach to obtain the solutions. As shown previously, the time-dependent variational approach is quite effective to study the dynamics of 3D BEC in trapping potential with conservative perturbations [18]. These autosoliton solutions can be considered as the nonlinear modes of such systems like solitons for the integrable NLSE [19]. Exact numerical simulations are also performed in the present paper, which confirms that, in the present case, the variational approach is a convenient and reliable approximation.

\section{THE MODEL AND THE VARIATIONAL APPROACH}

Let us describe the dynamics of a trapped Bose-Einstein condensate in the framework of the Gross-Pitaevskii equa- 
tion. The space-time dynamics of the condensate wave function can be analyzed by the GP equation in the mean-field approximation:

$$
\begin{aligned}
\mathrm{i} u_{t}+ & \Delta u-\left(\Omega^{2} r^{2}\right) u+\lambda_{2}|u|^{2} u+\lambda_{3}|u|^{4} u \\
& =\mathrm{i}\left(\gamma-\mu|u|^{2}-\xi|u|^{4}\right) u=R\left(u, u^{*}\right),
\end{aligned}
$$

where we use a standard simplified notation for the time derivative through a lower index $t . \Omega^{2} r^{2}$ is the trap harmonic potential and $\lambda_{2}$ and $\lambda_{3}$ are, respectively, the two- and threebody interaction parameters, where $\lambda_{2}\left(=a_{s}\right)$ is given by the $s$ - wave atomic scattering length. $\gamma, \mu$, and $\xi$ are positive defined coefficients related, respectively, to feeding, dipolar relaxation and three-body inelastic recombination parameters. We drop the explicit time and radial dependence of the functions, unless they are necessary for clarity.

In the present variational approach, for $u \equiv u(r, t)$, we use the Gaussian trial function [20]

$$
u=A(t) \exp \left(-\frac{r^{2}}{2 a^{2}(t)}+\mathrm{i} \frac{b(t) r^{2}}{2}+\mathrm{i} \phi(t)\right),
$$

where $A, a, b$, and $\phi$ are, respectively, the amplitude, width, chirp, and linear phase. We did not include the center-ofmass coordinate into the ansatz, because the dissipative and amplifying terms have no influence on it.

The variational approach is applied to the averaged Lagrangian of the conservative system

$$
L=\int \mathcal{L}(r, t) d^{3} r
$$

where the Lagrangian density, $\mathcal{L} \equiv \mathcal{L}(r, t)$, is given by

$$
\mathcal{L}=\frac{\mathrm{i}}{2}\left(u_{t} u^{*}-u_{t}^{*} u\right)-|\nabla u|^{2}+\frac{\lambda_{2}}{2}|u|^{4}+\frac{\lambda_{3}}{3}|u|^{6}-\Omega^{2} r^{2}|u|^{2} .
$$

Substituting the trial function (2) into Eqs. (4) and (3), we find the averaged Lagrangian in terms of the condensate wave-function parameters

$$
\begin{aligned}
L= & -\frac{\pi \sqrt{\pi}}{4} A^{2} a^{3}\left[3 a^{2} b_{t}+4 \phi_{t}+\frac{6}{a^{2}}\left(1+a^{4} b^{2}\right)-\frac{\lambda_{2}}{\sqrt{2}} A^{2}\right. \\
& \left.-\frac{4 \lambda_{3}}{9 \sqrt{3}} A^{4}+6 \Omega^{2} a^{2}\right] .
\end{aligned}
$$

We formally add $\mathcal{L}_{R}$ to Eq. (4), with the property that $\delta \mathcal{L}_{R} / \delta u^{*}=-R\left(u, u^{*}\right)$, where $R$ is on the right-hand side of Eq. (1). By applying the Euler-Lagrange equations to $\mathcal{L}^{\prime}$ $\equiv \mathcal{L}+\mathcal{L}_{R}$, with respect to $u^{*}$, we obtain

$$
\left[\frac{\partial \mathcal{L}^{\prime}}{\partial u^{*}}-\frac{d}{d t} \frac{\partial \mathcal{L}^{\prime}}{\partial u_{t}^{*}}\right]=\left[\frac{\partial \mathcal{L}}{\partial u^{*}}-\frac{d}{d t} \frac{\partial \mathcal{L}}{\partial u_{t}^{*}}\right]-R\left(u, u^{*}\right)=0,
$$

which leads to Eq. (1). (The conjugate equation is obtained in a similar way.)
The corresponding variational principle is given by

$$
\delta \int_{0}^{t} L^{\prime} d t=\delta \int_{0}^{t}\left(L+L_{R}\right) d t=0,
$$

where, as in Eq. (3), $L_{R}=\int d^{3} r \mathcal{L}_{R}$. Taking into account that for a small shift $\delta \eta$ of some variational parameter $\eta$, we have

$$
f(\eta+\delta \eta)=f(\eta)+\delta \eta \frac{\partial f}{\partial \eta},
$$

where $f \equiv f\left(u, u^{*}\right)=L$ or $L_{R}$, we obtain a system of equations for the variational parameters $\eta_{i}[20,21]$ :

$$
\frac{\partial L}{\partial \eta_{i}}-\frac{d}{d t} \frac{\partial L}{\partial \eta_{i t}}=\int d^{3} r\left[R \frac{\partial u^{*}}{\partial \eta_{i}}+R^{*} \frac{\partial u}{\partial \eta_{i}}\right],
$$

where Eq. (6) and its conjugate were used.

The substitution of Eqs. (2) and (5) into Eq. (9), yields the following system of ordinary differential equations (ODE's):

$$
\begin{gathered}
\frac{d\left(A^{2} a^{3}\right)}{d t}=2 \gamma A^{2} a^{3}-\frac{\mu}{\sqrt{2}} A^{4} a^{3}-\frac{2}{3 \sqrt{3}} \xi A^{6} a^{3}, \\
\frac{d\left(A^{2} a^{5}\right)}{d t}=4 A^{2} a^{5} b+2 \gamma A^{2} a^{5}-\frac{\mu}{2 \sqrt{2}} A^{4} a^{5}-\frac{2}{9 \sqrt{3}} \xi A^{6} a^{5} \\
\frac{d b}{d t}=\frac{2}{a^{4}}-2 b^{2}-2 \Omega^{2}-\frac{\lambda_{2} A^{2}}{2 \sqrt{2} a^{2}}-\frac{4 \lambda_{3} A^{4}}{9 \sqrt{3} a^{2}}, \\
\frac{d \phi}{d t}=-\frac{3}{a^{2}}+\frac{7}{8 \sqrt{2}} \lambda_{2} A^{2}+\frac{2}{3 \sqrt{3}} \lambda_{3} A^{4} .
\end{gathered}
$$

Equation (10a) can also be obtained from the modified form of the conservation law for the number of atoms $N$, where $N$ is given by

$$
N=\int|u|^{2} d^{3} r
$$

The other equations of the system (10) can be obtained by using higher moments, as shown in Appendix A. It is useful to rewrite the system using the notation $x=a^{2}, y=A^{2}$ :

$$
\begin{gathered}
x_{t}=4 x b+\frac{\mu}{2 \sqrt{2}} x y+\frac{4}{9 \sqrt{3}} \xi y^{2} x, \\
y_{t}=-6 y b+2 \gamma y-\frac{7 \mu}{4 \sqrt{2}} y^{2}-\frac{4}{3 \sqrt{3}} \xi y^{3}, \\
b_{t}=\frac{2}{x^{2}}-2 b^{2}-2 \Omega^{2}-\frac{\lambda_{2} y}{2 \sqrt{2} x}-\frac{4 \lambda_{3} y^{2}}{9 \sqrt{3} x} .
\end{gathered}
$$

This ODE system is the main result of this section. 


\section{ANALYSIS OF THE FIXED POINTS}

The autosoliton solution corresponds to the fixed points of the system. Recall the main properties of the autowave (dissipative) soliton. This solution has the form such as for the standard soliton, defined by the balance between amplification and nonlinear dissipative terms. As shown in the analysis of the one-dimensional case (the Pereira-Stenflo soliton), the solution is fixed by the parameters related to the amplification and dissipation with chirped phase. It does not depend on the initial conditions.

We restrict our analysis to $\lambda_{3}=0$. From the system given by Eq. (12), we can obtain the fixed points. Next we distinguish three cases:

(1) Case $\mu=0, \xi \neq 0$ :

In this case,

$$
y_{s 1}=\sqrt{\frac{3 \sqrt{3} \gamma}{\xi}}, \quad b_{s 1}=-\frac{1}{3} \gamma,
$$

and the width is

$$
x_{s 1}=-\frac{p_{1}}{2}+\sqrt{\frac{p_{1}^{2}}{4}+k_{1}},
$$

where

$$
p_{1}=\frac{\lambda_{2} y_{s 1}}{4 \sqrt{2}\left(\Omega^{2}+b_{s 1}^{2}\right)}, \quad k_{1}=\frac{1}{\Omega^{2}+b_{s 1}^{2}} .
$$

Let us consider that $p_{1} \gg k_{1}$. Then the solution is

$$
x_{s 1} \approx \frac{k_{1}}{p_{1}} \approx \frac{4 \sqrt{2} \Omega^{4}}{\lambda_{2} y_{s 1}} \text {. }
$$

(2) Case $\xi=0, \mu \neq 0$ :

The fixed points are

$$
y_{s 2}=\frac{2 \sqrt{2} \gamma}{\mu}, b_{s 2}=-\frac{\gamma}{4} .
$$

and the width is defined by the same expression as before Eq. (14)with $y_{s 2}, b_{s 2}$.

(3) Case $\mu \neq 0, \xi \neq 0$.

The fixed points are

$$
\begin{gathered}
y_{s 3}=-\frac{3 \sqrt{3} \mu}{4 \sqrt{2} \xi}+\sqrt{\frac{27 \mu^{2}}{32 \xi^{2}}+\frac{3 \sqrt{3} \gamma}{\xi},} \\
b_{s 3}=\frac{\mu}{24 \sqrt{2}} y_{s 3}-\frac{\gamma}{3}, x_{s 3}=-\frac{p_{3}}{2}+\sqrt{\frac{p_{3}^{2}}{2}+k_{3}},
\end{gathered}
$$

where

$$
p_{3}=\frac{\lambda_{2} y_{s 3}}{4 \sqrt{2}\left(\Omega^{2}+b_{s 3}^{2}\right)}, \quad k_{3}=\frac{1}{\Omega^{2}+b_{s 3}^{2}} .
$$

When $\mu=0(\xi \neq 0)$, we recover Eqs. (13) and (14). Alternatively, when $\xi \rightarrow 0(\mu \neq 0)$ we recover Eq. (16).

We now investigate the stability of the fixed points, in cases 1 and 2 , by using the linear stability analysis:

(1) Case $\mu=0, \xi \neq 0$ :

Let present the solutions of the system as $x=x_{s 1}+x_{1}, y$ $=y_{s 1}+y_{1}$, and $b=b_{s 1}+b_{1}$. The linearized system for corrections is

$$
\begin{gathered}
x_{1 t}=\frac{8}{9 \sqrt{3}} \xi y_{s 1} x_{s 1} y_{1}+4 x_{s 1} b_{1} \equiv-c_{2} y_{1}+c_{3} b_{1}, \\
y_{1 t}=-8 \gamma y_{1}-6 y_{s 1} b_{1} \equiv-d_{2} y_{1}-d_{3} b_{1}, \\
b_{1 t}=\frac{4}{x_{s 1}^{2}}\left(\frac{\lambda_{2} y_{s 1}}{8 \sqrt{2}}-\frac{1}{x_{s}}\right) x_{1}-\frac{\lambda_{2}}{2 \sqrt{2} x_{s 1}} y_{1}-4 b_{s 1} b_{1} \\
\equiv a_{1} x_{1}-a_{2} y_{1}-a_{3} b_{1} .
\end{gathered}
$$

With the solutions $x_{1}, y_{1}$ and $b_{1}$ having the same exponential behavior in time, given by $\sim e^{q t}$, we obtain the characteristic equation

$$
q^{3}+\alpha_{1} q^{2}-\alpha_{2} q-\alpha_{3}=0
$$

where

$$
\begin{aligned}
\alpha_{1} & \equiv\left(d_{2}+a_{3}\right)=\frac{20}{3} \gamma, \\
\alpha_{2} & \equiv\left(a_{1} c_{3}+a_{2} d_{3}-d_{2} a_{3}\right) \\
& =\frac{32}{3} \gamma^{2}+\frac{16}{x_{s 1}^{2}}\left(\frac{\lambda_{2} y_{s 1} x_{s 1}}{8 \sqrt{2}}-1\right)+\frac{3 \lambda_{2} y_{s 1}}{\sqrt{2} x_{s 1}}, \\
\alpha_{3} & \equiv a_{1}\left(d_{3} c_{2}+d_{2} c_{3}\right)=\frac{64 \gamma}{x_{s 1}^{2}}\left(\frac{\lambda_{2} y_{s 1} x_{s 1}}{8 \sqrt{2}}-1\right) .
\end{aligned}
$$

The roots with $\operatorname{Re}(q)>0$ correspond to the unstable solutions.

Without loss of generality, in our dimensionless NLSE we can scale the parameters as $\lambda_{2}=1$ and $\Omega=1$ [13]. The diagram of stability, according to the solutions of Eq. (20), is presented in Fig. 1. The diagram clearly shows that, when $\gamma>1.84 \xi$, the system is unstable. If $\gamma \gg \xi$, the system enters the collapsing process, shown in Ref. [16] to be chaotic. If $\gamma$ is decreased (or $\xi$ increased) the system will eventually achieve a stable region where the formation of an autosoliton is possible.

(2) Case $\xi=0, \mu \neq 0$ :

Analogously, as in case 1 , we present the solutions of the system as $x=x_{s 2}+x_{2}, y=y_{s 2}+y_{2}$, and $b=b_{s 2}+b_{2}$. The linearized system for corrections is given by

$$
x_{2 t}=\frac{\mu x_{s 2}}{2 \sqrt{2}} y_{2}+4 x_{s 2} b_{2}
$$




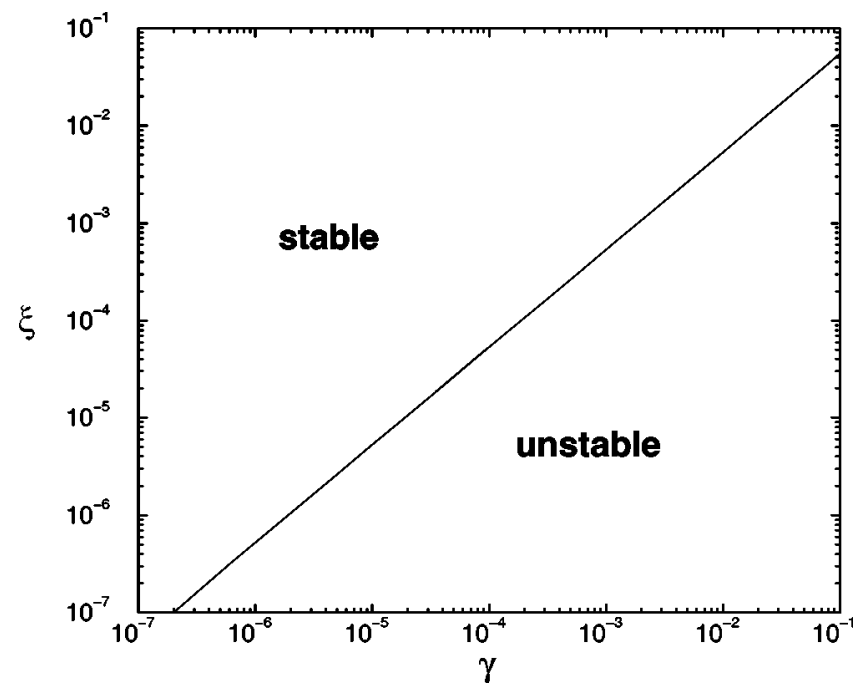

FIG. 1. Gaussian variational analysis of stability of the fixed points for the Gross-Pitaevskii equation including feeding $(\gamma)$ and three-body losses $(\xi)$.

$$
\begin{gathered}
y_{2 t}=-\frac{7}{2} \gamma y_{2}-\frac{12 \sqrt{2} \gamma}{\mu} b_{2}, \\
b_{2 t}=\frac{4}{x_{s 2}^{3}}\left(\frac{\lambda_{2} \gamma}{4 \mu} x_{s 2}-1\right) x_{2}-\frac{\lambda_{2}}{2 \sqrt{2} x_{s 2}} y_{2}-4 b_{s 2} b_{2} .
\end{gathered}
$$

The system has the same form as in Eq. (19). Correspondingly, the characteristic equation is given by

$$
q^{3}+\beta_{1} q^{2}-\beta_{2} q-\beta_{3}=0
$$

where

$$
\begin{gathered}
\beta_{1}=\frac{5}{2} \gamma, \quad \beta_{2}=\frac{7}{2} \gamma^{2}+\frac{16}{x_{s 2}^{2}}\left(\frac{\lambda_{2} \gamma x_{s 2}}{4 \mu}-1\right)+\frac{6 \lambda_{2} \gamma}{x_{s 2} \mu}, \\
\beta_{3}=\frac{32 \gamma}{x_{s 2}^{2}}\left(\frac{\lambda_{2} \gamma x_{s 2}}{4 \mu}-1\right) .
\end{gathered}
$$

With the same scaling used in case $1\left(\lambda_{2}=1\right.$ and $\Omega$ $=1$ ), and with the above solutions of Eq. (23), we obtain the diagram of stability, shown in Fig. 2. The diagram clearly shows that, when $\gamma>0.53 \mu$, the system is unstable. In analogy with the previous case, if $\gamma$ is decreased (or $\mu$ increased), the system will eventually achieve a stable region where the formation of the autosoliton is possible.

\section{NUMERICAL SIMULATIONS AND DISCUSSIONS}

We did a series of time-dependent simulations of the system within the Gaussian variational approach, using Eq. (12), and also by performing exact numerical calculations with Eq. (1). In our numerical calculations, we have used the finitedifference Crank-Nicolson algorithm. The exact initial wave functions were used following the prescription given in Ref. [22]. Next we present simulations in a range of parameters

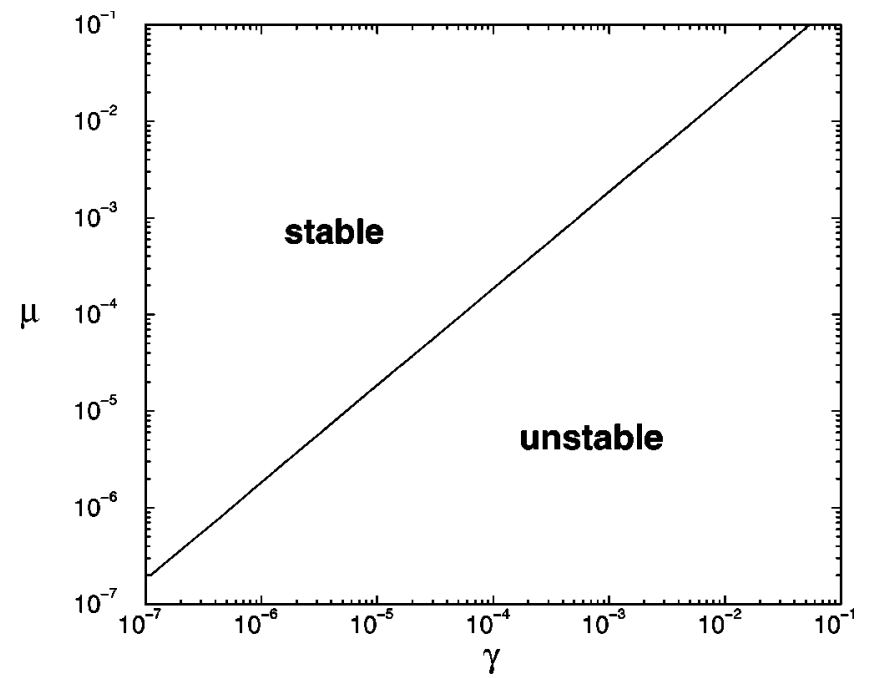

FIG. 2. Gaussian variational analysis of stability of the fixed points for the Gross-Pitaevskii equation including feeding $(\gamma)$ and two-body losses $(\mu)$.

that lead to long-time stable autosolitons. We obtain results with autosolitonic solutions for a class of parameters that are near the realistic ones, as indicated by ${ }^{7} \mathrm{Li}$ experiments in BEC.

In Fig. 3, for $\gamma=10^{-3}$ and $\xi=10^{-3}$, we show the time evolution of the number of atoms, in terms of the maximum critical number for stability, $N_{c}$. The formation of the autosoliton is demonstrated either by Gaussian variational approach or by exact numerical calculations. There is a remarkable agreement between both approaches. Note that the number of atoms does not depend on the initial conditions, but is related to the equilibration of the feeding and dissipa-

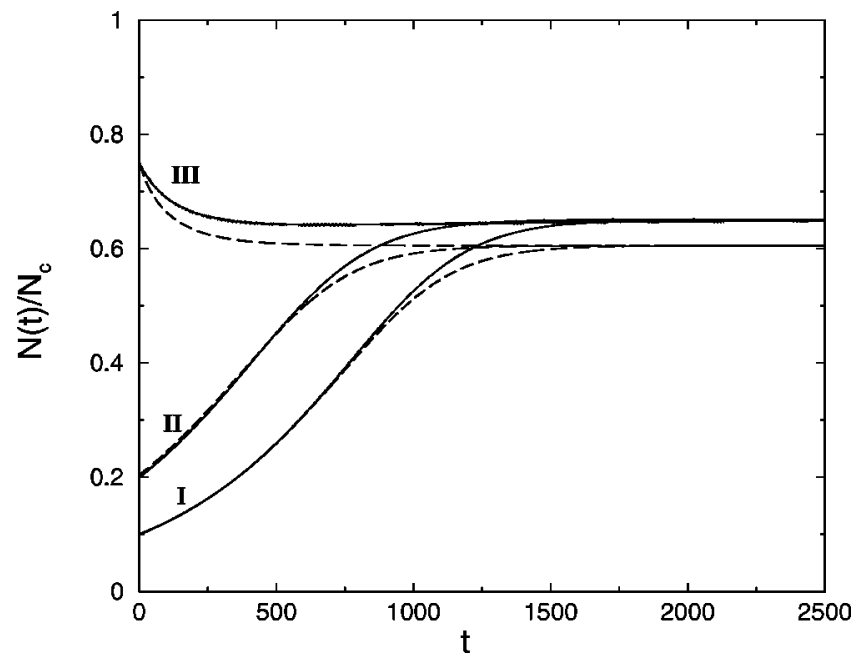

FIG. 3. Evolution of the number of atoms $N$ in the GrossPitaevskii equation with feeding parameter $\gamma=10^{-3}$ and three-body dissipation parameter $\xi=10^{-3}(\mu=0)$. The results are represented by solid lines for the variational approach, and by dashed lines for the exact numerical calculations. Cases I, II, and III correspond, respectively, to the initial conditions $N(t=0) / N_{c}=0.1,0.2$ and 0.75, where $N_{c}$ is the maximum critical number for stability. $t$ is given in units of $2 / \Omega$, where $\Omega$ is the trap frequency. 


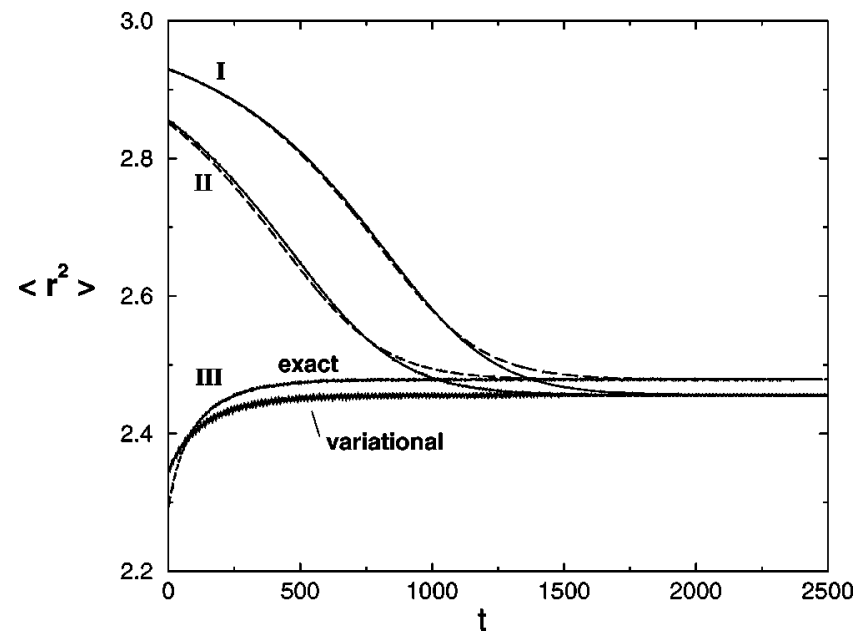

FIG. 4. Evolution of the mean-square radius, Eq. (25), in the Gross-Pitaevskii equation. The same parameters and conventions given in Fig. 3 were used. The mean-square radius is given in units of $\hbar /(2 \mathrm{~m} \Omega)$.

tion. The variational approach results give a little higher number of atoms than the exact calculations.

Corresponding to the results in Fig. 3, we show in Fig. 4 the results for the time evolution of the mean-square radius, where

$$
\left\langle r^{2}\right\rangle=\int r^{2}|u|^{2} d^{3} r
$$

In this case, the variational approach results are a bit lower than the ones obtained by exact calculations.

In analogy with the case that $\mu=0$, represented in Figs. 3 and 4 , we also present results for the case where the threebody dissipation parameter $(\xi)$ is zero. The results obtained for the time evolution of the number of atoms and the meansquare radius are, respectively, shown in Figs. 5 and 6, for

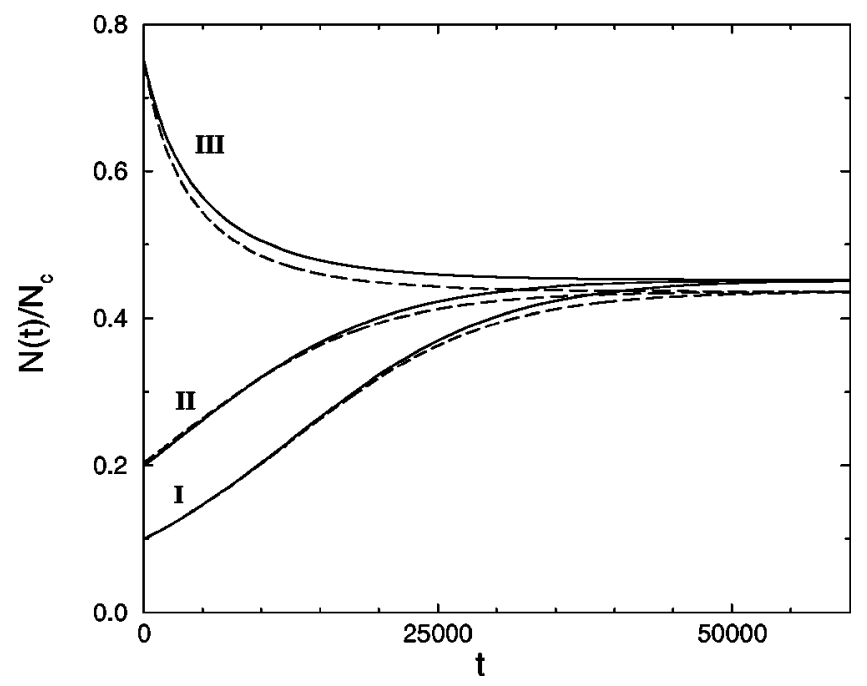

FIG. 5. Evolution of the number of atoms in the GrossPitaevskii equation with feeding $\gamma=5 \times 10^{-5}$ and two-body dissipation parameter $\mu=10^{-4}(\xi=0)$. The initial conditions and conventions are the same as in Fig. 3.

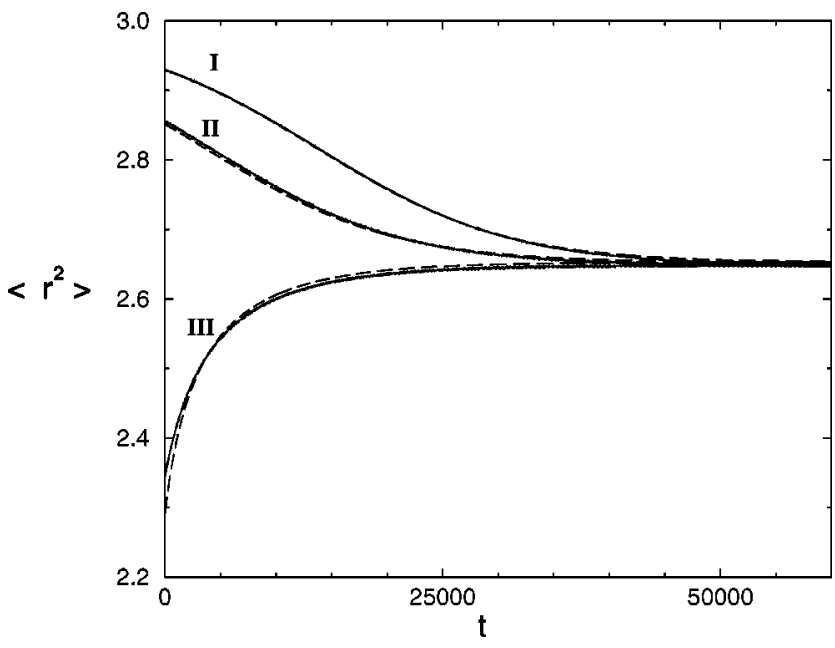

FIG. 6. Evolution of the mean-square radius in the GrossPitaevskii equation. The parameters and conventions are the same as in Fig. 5. The mean-square radius is given in units of $\hbar /(2 \mathrm{~m} \Omega)$.

$\gamma=5 \times 10^{-5}$ and $\mu=10^{-4}$. The formation of the autosoliton is also demonstrated either by Gaussian variational approach or by exact numerical calculations. The remarkable agreement between both approaches, already observed in the case where $\mu=0$ (Figs. 3 and 4), also applies to this case where $\xi=0$, as the number of atoms do not depend on the initial conditions.

In ${ }^{7} \mathrm{Li}$ experiment the feeding parameter can be indirectly inferred from measurements done by the Rice group [14] and will correspond to an average rate of about 600 atoms/s [23]. The two- and three-body losses were also measured [24] and estimated [25], giving atom loss rates of about 2 $\times 10^{-14} \mathrm{~cm}^{3} / \mathrm{s}$ and $\sim 10^{-28} \mathrm{~cm}^{6} / \mathrm{s}$. These rates were measured for noncondensed atoms. For condensed atoms they must be divided by factors of 2 ! and 3!, respectively [26]. With a scaled equation, such that $\lambda_{2}=1$ and $\Omega=1$, we have for condensed atoms $\gamma \sim 10^{-3}, \mu \sim 10^{-4}$, and $\xi \sim 10^{-6}$. Considering this magnitudes, the autosoliton can possibly be observed experimentally in ${ }^{7} \mathrm{Li}$, either by results with decreasing $\gamma$ due to losses, or by increasing the dissipation rate due to other mechanism such as the Feshbach resonances [27]. In the case of diminishing $\gamma$, the autosoliton formation is more likely determined by dipolar relaxation rather than three-body recombination losses.

\section{CONCLUSION}

In this paper we studied the possibility of existence of autosolitons in trapped 3D BEC in the presence of two- and three-body inelastic processes: dipolar relaxation and threebody recombination. Using the time-dependent variational approach for the nonconservative 3D Gross-Pitaevskii equation, we derived expressions for the parameters of the autosoliton and checked their stability. The results obtained by using the present time-dependent Gaussian variational approach, in the NLSE with atomic feeding and nonlinear dissipative terms, show a remarkable agreement with exact numerical calculations when the parameters were such that stabilization was achieved. We should note that, stabilization can also be numerically observed in the model given in Ref. 
[13], when $\gamma$ is decreased in time, but their parameters are far from the realistic ones and dipolar relaxation was neglected. Such stabilization verified in Ref. [13] has not been recognized as a manifestation of the autosoliton, a characterization that we are pointing out in the present paper.

We have shown that the transition from unstable (collapsing) to stable point (autosoliton) solely depends on the magnitude of the parameters. Also the present paper includes non-negligible two-body dissipative effects that model the dipolar relaxation losses, and that can be associated with values measured in ultracold ${ }^{7} \mathrm{Li}$ [24]. In the case of decreasing $\gamma$ due to collapsing and losses, the autosoliton is more likely to be formed first due to dipolar relaxation rather than by three-body recombination processes. These results can be relevant in current experiments with negative scattering length and possibly display new phenomenon of PereiraStenflo type autosoliton formation in Bose condensates. We believe that such phenomenon is already occurring in the long-time behavior in the actual experiments with ${ }^{7} \mathrm{Li}$ [14] $(\Omega \sim 2 \pi \times 140 \mathrm{~Hz})$, since for longer times $(\sim 60 \mathrm{~s})$ the maximum number of atoms $\left(N_{c} \sim 1300\right.$ atoms $)$ is considerably reduced, as expected in our simulations. We hope that experiments with direct observation of the evolution of the condensate can clarify this matter.

\section{ACKNOWLEDGMENTS}

A.G. is grateful to Professor R.G. Hulet for useful discussions. F.K.A. is grateful to Instituto de Física Teórica for the hospitality, and also to partial support of US CRDF (Award No. ZM2-2095). We thank Fundação de Amparo à Pesquisa do Estado de São Paulo (FAPESP) for partial financial support. L.T. also thanks partial support from Conselho Nacional de Desenvolvimento Científico e Tecnológico (CNPq). V.S.F. thanks support received from Coordenação de Aperfeiçoamento de Pessoal de Nível Superior (CAPES).

\section{APPENDIX}

The first equation of the system (10) can be obtained by calculating the rate of change of the number of atoms as

$$
\frac{d N}{d t}=\frac{d}{d t} \int d^{3} r|u|^{2}=\int\left(u_{t}^{*} u+u^{*} u_{t}\right) d^{3} r .
$$

Taking $u_{t}$ from Eq. (1) and substituting in Eq. (A1) we obtain the modified form of the conservation law for the number of atoms [29]

$$
\frac{d N}{d t}=2 \gamma N-2 \mu \int|u|^{4} d^{3} r-2 \xi \int|u|^{6} d^{3} r .
$$

Substituting into this equation the Gaussian trial function (2) we obtain the first equation of the system given in Eq. (10).

We have derived the Eqs. (10b) and (10c) by the moments method. Eq. (10b) can be derived by calculating

$$
\frac{d\left\langle r^{2}\right\rangle}{d t}=\int\left(u_{t}^{*} r^{2} u+u^{*} r^{2} u_{t}\right) d^{3} r .
$$

Substituting the $u_{t}$ from expression (1) and applying the commutation rules, we get [28]

$$
\begin{aligned}
\frac{d\left\langle r^{2}\right\rangle}{d t}= & 4 \operatorname{Im} \int u * \vec{r} \cdot(\nabla u) d^{3} r+2 \gamma \int|u|^{2} r^{2} d^{3} r \\
& -2 \mu \int|u|^{4} r^{2} d^{3} r-2 \xi \int|u|^{6} r^{2} d^{3} r .
\end{aligned}
$$

The substitution of the Gaussian ansatz [Eq. (2)] in both sides of this expression results directly in Eq. (10b).

Equation (10c) can be derived analogously by showing that

$$
\begin{aligned}
\frac{d\left\langle p^{2}\right\rangle}{d t}= & -4 \Omega^{2} \operatorname{Im} \int u^{*} \vec{r} \cdot(\boldsymbol{\nabla} u) d^{3} r \\
& +2 \operatorname{Im} \int\left(\boldsymbol{\nabla} u^{*}\right) \cdot \boldsymbol{\nabla}\left(V_{1} u\right) d^{3} r \\
& +2 \operatorname{Re} \int\left(\boldsymbol{\nabla} u^{*}\right) \cdot \boldsymbol{\nabla}\left(V_{2} u\right) d^{3} r,
\end{aligned}
$$

where $V_{1}=-\lambda_{2}|u|^{2}-\lambda_{3}|u|^{4}$ and $V_{2}=\gamma-\mu|u|^{2}-\xi|u|^{4}$. Substituting the Gaussian ansatz [Eq. (2)] in both sides of Eq. (A5) and using results (10a) and (10b), there is overall cancellation of the feeding and dissipative terms and we finally get Eq. (10c).
[1] N.R. Pereira and L. Stenflo, Phys. Fluids 20, 1733 (1977).

[2] G. Agrawal, Nonlinear Fiber Optics (Academic, New York, 1994).

[3] F.Kh. Abdullaev, S.A. Darmanyan, and P.K. Khabibullaev, Optical Solitons (Springer-Verlag, Heidelberg, 1993).

[4] M.V. Fabrikant, Wave Motion 2, 355 (1980).

[5] D. Anderson, F. Cattani, and M. Lisak, Phys. Scr. T82, 32 (1999).

[6] F.Kh. Abdullaev, Theory of Solitons in Inhomogeneous Media (Wiley, Chichester, 1994).

[7] F.Kh. Abdullaev, S.A. Darmanyan, and M.R. Djumaev, Izv.AN UzSSR 6, 34 (1986); Phys. Lett. A 141, 423 (1989).
[8] R. Dum, J.I. Cirac, M. Lewenstein, and P. Zoller, Phys. Rev. Lett. 80, 2972 (1998).

[9] H. Michinel, V.M. Perez-Garcia, and R. de la Fuente, Phys. Rev. A 60, 1513 (1999).

[10] J. Denschlag et al., Science 287, 97 (2000).

[11] A. Gammal, T. Frederico, L. Tomio, and F.Kh. Abdullaev, Phys. Lett. A 267, 305 (2000).

[12] L. Bergé, T.J. Alexander, and Y. Kivshar, Phys. Rev. A 62, 023607 (2000).

[13] Y. Kagan, A.E. Muryshev, and G.V. Shlyapnikov, Phys. Rev. Lett. 81, 933 (1998).

[14] C.A. Sackett, J.M. Gerton, M. Welling, and R.G. Hulet, Phys. 
Rev. Lett. 82, 876 (1999).

[15] C.A. Sackett, H.T.C. Stoof, and R.G. Hulet, Phys. Rev. Lett. 80, 2031 (1998).

[16] A. Gammal, T. Frederico, L. Tomio, and P. Chomaz, Phys. Rev. A 61, 051602(R) (2000); V.S. Filho, A. Gammal, L. Tomio, and T. Frederico, ibid. 62, 033605 (2000).

[17] D.J. Kaup and B.A. Malomed, Physica D 87, 155 (1995).

[18] J.J.G. Ripoll and V.M. Perez-Garcia, Phys. Rev. A 59, 2220 (1999).

[19] N. Akhmediev and A. Ankiewicz, in Spatial Solitons 1, edited by S. Trillo and W. E. Toruellas (Springer-Verlag, Berlin, 2000).

[20] A. Bondeson, M. Lisak, and D. Anderson, Phys. Scr. 20, 479 (1979).

[21] A.I. Maǐmistov, Zh. Éksp. Teor. Fiz. 104, 3620 (1993) [JETP 77, 727 (1993)].
[22] A. Gammal, T. Frederico, and L. Tomio, Phys. Rev. E 60, 2421 (1999).

[23] R.G. Hulet (private communication).

[24] J.M. Gerton, C.A. Sackett, B.J. Frew, and R.G. Hulet, Phys. Rev. A 59, 1514 (1999).

[25] A.J. Moerdijk, H.M.J.M. Boesten, and B.J. Verhaar, Phys. Rev. A 53, 916 (1996); A.J. Moerdijk and B. Verhaar, ibid. 53, R19 (1996).

[26] Yu. Kagan, B.V. Svistunov, and G.V. Shlyapnikov, Pis'ma Zh. Éksp. Teor. Fiz. 42, 169 (1985) [JETP Lett. 42, 209 (1985)]; E.A. Burt et al., Phys. Rev. Lett. 79, 337 (1997).

[27] S.L. Cornish et al., Phys. Rev. Lett. 85, 1795 (2000).

[28] T. Tsurumi and M. Wadati, J. Phys. Soc. Jpn. 68, 1531 (1999).

[29] A.S. Parkins and D.F. Walls, Phys. Rep. 303, 1 (1998), and references therein. 